\title{
ON DISTAL AND EQUICONTINUOUS COMPACT RIGHT TOPOLOGICAL GROUPS
}

\author{
PAUL MILNES \\ University of Western Ontario \\ London, Ontario N6A 5B7, Canada
}

(Received June 2, 1992)

\begin{abstract}
W. Ruppert has studied, and given examples of, compact left topological groups for which the left translation flow $\left(\lambda_{G}, G\right)$ is equicontinuous. Recently, we considered an analogous distal condition that applies to the groups of dynamical type; for these the topological centre is dense, so the translation flow is equicontinuous only in the trivial case when $G$ is topological. In the present paper, we continue this work, exhibiting new characterizations of equicontinuity and distality of $\left(\lambda_{G}, G\right)$; we also discuss examples and study some related matters.
\end{abstract}

KEY WORDS AND PHRASES. Compact group, one-sided continuity, equicontinuous flow, distal flow.

1991 AMS SUBJECT CLASSIFICATION CODES. 22A05, 54H20.

\section{INTRODUCTION.}

For a compact left topological group $G$, the minimality of the left translation flow $\left(\lambda_{G}, G\right)$ is obvious; in fact, one might find it a little surprising that $\left(\lambda_{G}, G\right)$ is not always distal. Perhaps more surprising is that $\left(\lambda_{G}, G\right)$ can be equicontinuous (for non-topological $G$ ). W. Ruppert [16] studied this last case, and along with other interesting results and examples, his paper includes the conclusion that the topological centre

$$
\mathfrak{R}(G):=\{s \in G \mid t \mapsto t s, G \rightarrow G, \text { is continuous }\}
$$

is closed in $G$ for this situation. Since compact left topological groups coming from distal flows are characterized by having dense topological centres, they cannot yield equicontinuous $\left(\lambda_{G}, G\right)$ (except in the "trivial" situation, where $G$ is a topological group); however, for compact left topological groups in general (including those with dense topological centres), we determined in [11] that distality of $\left(\lambda_{G}, G\right)$ is an interesting property to consider, and found criteria for it analogous to criteria of Ruppert for equicontinuity. In the present paper, we find new criteria for distality and equicontinuity of $\left(\lambda_{G}, G\right)$ in terms of an analysis of $\mathcal{C}(G)$. The well-developed theory of function spaces on semitopological semigroups $[6,4,14,5$, for example] does not apply directly to $G$, because $G$ is not semitopological. However, we prove that the containment $\mathcal{C}(G) \subset \mathcal{A P}\left(G_{d}\right)$ is equivalent to to equicontinuity of $\left(\lambda_{G}, G\right)$, and have an analogous conclusion in the distal situation (Theorem 3 ). We have yet another criterion for equicontinuity in terms of translation operators on $\mathcal{C}(G)$ (Theorem 8), and we study $\mathcal{C}(G) \cap \mathcal{A P}\left(G_{d}\right)$ in the setting 
where the translation flow is not equicontinuous, connecting this subalgebra to the structure theorem for compact groups with one-sided continuity and dense topological centre (Theorem $6)$.

\section{PRELIMINARIES.}

A flow $(S, X)$ consists of a compact Hausdorff space $X$ and a group $S$ with identity $e$ acting on it on the left (as in [15]): each $s \in S$ determines a homeomorphism $x \mapsto s x$ of $X$ and the conditions $e x=x$ and $s(t(x))=(s t) x$ for all $s, t \in S$ and $x \in X$ are satisfied. So, $S$ dctermines a subgroup (denoted here also by $S$ ) of the semigroup $X^{X}$ of all transformations of $X$. The closure $S^{-}$of $S$ in $X^{X}$ is a subsemigroup of $X^{X}$ called the enveloping semigroup of the flow. With the relative topology from $X^{X}, S^{-}$is a compact right topological semigroup, i.e., for all $\eta \in S^{-}$, right multiplication by $\eta, \nu \mapsto \nu \eta, S^{-} \rightarrow S^{-}$, is continuous. The set

$$
\Lambda\left(S^{-}\right):=\left\{\eta \in S^{-} \mid \nu \mapsto \eta \nu, S^{-} \rightarrow S^{-} \text {is continuous }\right\}
$$

is called the topological centre of $S^{-}$; here $S \subset \Lambda\left(S^{-}\right)$, so $\Lambda\left(S^{-}\right)$is dense in $S^{-}$. The flow is equicontinuous if the transformations $S$ are equicontinuous with respect to the unique uniformity of the compact space $X$, and distal if $s_{\alpha} x_{1} \rightarrow x_{0}$ and $s_{\alpha} x_{2} \rightarrow x_{0}$ for net $\left\{s_{\alpha}\right\} \subset S$ and $x_{0}, x_{1}, x_{2} \in X$ always implies $x_{1}=x_{2}$. We quote a beautiful theorem of Ellis [7, or 8].

1. THEOREM. A flow $(S, X)$ is distal if and only if its enveloping semigroup $S^{-}$is a group (i.e., a subgroup of $X^{X}$ ).

For a distal flow $(S, X)$, the compact right topological group $G:=S^{-}$is called the Ellis group of the flow. There is a powerful structure theorem for compact groups $G$ that come from topological dynamics like this (i.e., have dense topological centres); it developed over an extended period $[9,8,15]$, the claim about normality of each subgroup $L_{\xi}$ in $G$ (rather than just in $L_{\xi-1}$ for successor ordinals $\xi$ ) having been established only recently $[12,13]$.

\section{THE FURSTENBERG - ELLIS - NAMIOKA STRUCTURE THEOREM.}

Let $G$ be a compact right topological group with $\Lambda(G)$ dense in $G$. Then $G$ has a system of subgroups

$$
\left\{L_{\xi} \mid 0 \leq \xi \leq \xi_{0}\right\}
$$

ordered by the set of ordinals less than or equal to an ordinal $\xi_{0}$ and satisfying

(i) each $L_{\xi}$ is a closed normal subgroup of $G, L_{0}=G$, and $L_{\xi_{0}}=\{e\}$;

(ii) for $\xi<\xi_{0}, L_{\xi} \supset L_{\xi+1}$, and the function

$$
\left(s L_{\xi+1}, t L_{\xi+1}\right) \mapsto s t L_{\xi+1}, G / L_{\xi+1} \times L_{\xi} / L_{\xi+1} \rightarrow G / L_{\xi+1}
$$

is continuous for the quotient topologies; and

(iii) for each limit ordinal $\xi \leq \xi_{0}, L_{\xi}=\bigcap_{\eta<\xi} L_{\eta}$.

A consequence of Theorem 2 is the existence of Haar measure $\mu$ for compact right topological groups $G$ with dense topological centres; $\mu$ is the unique probability measure on $G$ that is invariant under all right translations and all continuous left translations $[12,13]$.

The reader will have noticed that we discussed only left topological groups in the Abstract and Introduction, yet here we are defining right topological groups and semigroups. As will be 
made clear in the next section, we do need to deal also with compact left topological groups $G$, for which the left multiplications $t \mapsto \lambda_{s}(t):=s t, G \rightarrow G$, are all continuous, so we get the left translation flow $\left(\lambda_{G}, G\right)$; now

$$
\mathfrak{R}(G):=\{s \in G \mid t \mapsto t s, G \rightarrow G, \text { is continuous }\}
$$

is the topological centre of $G$. Such a group $G$ is called distal, or equicontinuous, if the flow $\left(\lambda_{G}, G\right)$ is distal or equicontinuous. Analogously, a compact right topological group is called distal, or equicontinuous, if the right translation flow (as in Theorem 8 below) is distal or equicontinuous. If a group $G$ starts out with a compact topology, $\mathcal{C}(G)$ denotes the $C^{*}$-algebra of continuous complex-valued functions on $G$, and $G_{d}$ denotes the same algebraic group with the discrete topology. We shall be considering some function spaces on $G_{d}$, and denote by $R_{s}$ and $L_{t}$ the right and left translation operators, respectively, on $l^{\infty}\left(G_{d}\right)$,

$$
R_{s} f(t)=f(t s)=L_{t} f(s)
$$

Let $\mathcal{M} \mathcal{I N}\left(G_{d}\right), \mathcal{D}\left(G_{d}\right)$, and $\mathcal{A} \mathcal{P}\left(G_{d}\right)$ be the spaces of minimal, distal and almost periodic functions on $G_{d}$, respectively. These are defined in terms of the pointwise closure $R_{G} f^{-}$of the right orbit of $f \in l^{\infty}\left(G_{d}\right) ; G_{d}$ acts on $R_{G} f^{-}$by right translation, and $f$ is minimal, distal, or almost periodic, if the resulting flow $\left(R_{G}, R_{G} f^{-}\right)$is minimal, distal, or equicontinuous. $\mathcal{D}\left(G_{d}\right)$ and $\mathcal{A P}\left(G_{d}\right)$ are left $m$-introverted subalgebras of $l^{\infty}\left(G_{d}\right)$, and the compactifications $G_{d}^{\mathcal{D}}$ and $G_{d}{ }^{\mathcal{A P}}$ of $G_{d}$ associated with them (which we view as the spectra of $\mathcal{D}\left(G_{d}\right)$ and $\mathcal{A P}\left(G_{d}\right)$, respectively) are the universal compact right topological group compactification and the universal compact topological group compactification of $G_{d}$, respectively. See [5] for more details on these matters.

\section{DISTALITY AND EQUICONTINUITY OF THE LEFT TRANSLATION FLOW $\left(\lambda_{G}, G\right)$.}

The results we want to present now involve a compact group with one-sided continuity and its translation flow, whose enveloping semigroup may have one-sided continuity only on the other side. Clearly, the only difference made by starting with continuity on one side, rather than on the other, is in the notation. Accordingly, we start in the setting where the notation feels the most familiar, with a compact left topological group (as did Ruppert [16]). For such a group $G$, the left translation flow $\left(\lambda_{G}, G\right)$ is always minimal, since $\lambda_{G} s=G$ for all $s \in G$. Similarly, $\mathcal{C}(G) \subset \mathcal{M I N}\left(G_{d}\right)$, because of the following easy consequence of the continuity of left multiplication: if $f \in \mathcal{C}(G)$ and $l^{\infty}\left(G_{d}\right)$ has the topology $p$ of pointwise convergence on $G$, then the function $s \mapsto R_{s} f, G \rightarrow l^{\infty}\left(G_{d}\right)$, maps $G$ continuously onto the right orbit $R_{G} f$ of $f$, which is therefore $p$-closed. Note that right translates of members of $\mathcal{C}(G)$ need not be continuous. The compact left topological group $G=\mathbf{T}^{\mathbf{T}} \times \mathbf{T}$, with multiplication

$$
\left(h^{\prime}, w^{\prime}\right)(h, w)=\left(h^{\prime} L_{w^{\prime}} h, w^{\prime} w\right)
$$

is not distal [11] (i.e., has flow $\left(\lambda_{G}, G\right)$ that is not distal), but $\left(\lambda_{G}, G\right)$ is of course minimal, as shown above. We are unable to identify the enveloping semigroup $\lambda_{G}{ }^{-} \subset G^{G}$ beyond saying that it is probably a fairly complicated subsemigroup of $X^{X} \times \mathbf{T}$, where $X:=\mathbf{T}^{\mathbf{T}}$. However, 
the group $G=\mathbb{T} \times\{ \pm 1\}$, as in Example 7.2 below, also is not distal [11]; in this case, we are able to identify $\lambda_{G}{ }^{-} \subset G^{G}$ as a "six-circle" semigroup, which we believe has interesting applications. ( $\lambda_{G}{ }^{-}$is a more elaborate version of the "three-circle" semigroup in [3].) The next theorem shows that we can sometimes place $\mathcal{C}(G)$ in a subalgebra of $l^{\infty}\left(G_{d}\right)$ that is much smaller than $\mathcal{M} \mathcal{I N}\left(G_{d}\right)$. Another condition equivalent to equicontinuity and way of arriving at the compact topological group $\lambda_{G}{ }^{-} \subset G^{G}$, when $G$ is equicontinuous (i.e., when $\left(\lambda_{G}, G\right)$ is equicontinuous), are discussed below; see Example 4(b) and Theorem 8.

3. THEOREM. Let $G$ be a compact left topological group. Then $G$ is

(i) distal if and only if $\mathcal{C}(G) \subset \mathcal{D}\left(G_{d}\right)$, and

(ii) equicontinuous if and only if $\mathcal{C}(G) \subset \mathcal{A P}\left(G_{d}\right)$.

PROOF. (i) Suppose that $\left(\lambda_{G}, G\right)$ is distal and that $f \in \mathcal{C}(G)$. Since the map $s \mapsto R_{s} f$ is a continuous homomorphism of the flow $\left(\lambda_{G}, G\right)$ onto the right translation flow $\left(R_{G}, R_{G} f^{-}\right)$(as above), the latter flow is also distal [8; Corollary 5.7], so $f \in \mathcal{D}\left(G_{d}\right)$.

Conversely, if $\mathcal{C}(G) \subset \mathcal{D}\left(G_{d}\right)$ and $\lim _{\alpha} s_{\alpha} t=\lim _{\alpha} s_{\alpha} t^{\prime}$ for some $t, t^{\prime} \in G$ and net $\left\{s_{\alpha}\right\} \subset G$, take an $f \in \mathcal{C}(G)$. Then

$$
\lim _{\alpha} R_{s_{\alpha}}\left(R_{t} f\right)=\lim _{\alpha} R_{s_{\alpha}}\left(R_{t^{\prime}} f\right),
$$

which implies that $R_{t} f=R_{t^{\prime}} f$, since $\left(R_{G}, R_{G} f^{-}\right)$is distal; so $f(t)=f\left(t^{\prime}\right)$. Because $f \in \mathcal{C}(G)$ was arbitrary, we have $t=t^{\prime}$.

(ii) If $\left(\lambda_{G}, G\right)$ is equicontinuous, we proceed as in (i) and conclude that $\left(R_{G}, R_{G} f^{-}\right)$, the homomorphic image of $\left(\lambda_{G}, G\right)$, is as well [8; Proposition 4.7], so $f \in \mathcal{A P}\left(G_{d}\right)$. Conversely, if $\mathcal{C}(G) \subset \mathcal{A P}\left(G_{d}\right)$, we have the natural map $\psi$ of $G$ into the almost periodic compactification $G_{d}{ }^{\mathcal{A P}}$ of $G_{d}$, and the flow $\left(G, G_{d}{ }^{\mathcal{A P}}\right),(s, \mu) \mapsto \psi(s) \mu$, is equicontinuous. Identifying $G$ with the spectrum of $\mathcal{C}(G)$, we note that the restriction mapping $\delta: G_{d} \mathcal{A P} \rightarrow G$ is a continuous homomorphism of $\left(G, G_{d}{ }^{A \mathcal{P}}\right)$ onto $\left(\lambda_{G}, G\right)$ (i.e., $\left.\delta(\psi(s) \mu)=s \delta(\mu)\right)$, which is therefore also equicontinuous [8; Proposition 4.7].

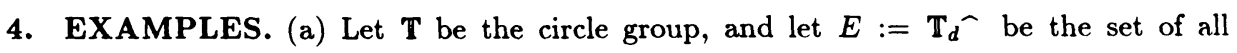
endomorphisms of $\mathbf{T}$. ( $E \cong \mathbf{Z}^{\mathcal{A P}}$, the almost periodic compactification of the integers $\mathbf{Z}[1$, or 5; 4.3.18].) Then $G=\mathbb{T} \times E$ with multiplication

$$
\left(w^{\prime}, h^{\prime}\right)(w, h)=\left(w^{\prime} w h \circ h^{\prime}\left(e^{2 i}\right), h^{\prime} h\right)
$$

is a distal compact left topological group [11], as may be readily verified; so, $\mathcal{C}(G) \subset \mathcal{D}\left(G_{d}\right)$. $f(w, h)=w$ defines a continuous function $f$ on $G$, a right translate of which, $R_{(w, h)} f$, is continuous if and only if

$$
(w, h) \in \Lambda(G)=\left\{(w, h) \mid h=()^{n} \text { for some } n \in \mathbf{Z}\right\}
$$

$\left(()^{n}: \mathbb{T} \rightarrow \mathbf{T}\right.$ being the character $\left.w \mapsto w^{n}\right)$; so, $\mathcal{C}(G) \cap R_{\{1\} \times E} f$ is countable, while the cardinality of $R_{\{1\} \times E} f$ is at least $2^{c}$. Thus, $\mathcal{C}(G)$ is not right translation invariant, and so is not a left $m$-introverted subalgebra of $l^{\infty}\left(G_{d}\right)$ [5]. Although it is left translation invariant, pointwise limits of members of $L_{G} f$ need not be continuous for $f \in \mathcal{C}(G) ; \mathcal{C}(G)$ is also not right $m$-introverted $[5 ; 2.2$, for example]. Right $m$-introverted subalgebras associated with the left topological compactification $G$ are the algebras of restrictions of members of $\mathcal{C}(G)$ to $\Lambda(G)$, or 
to its dense subgroup $\mathbf{Z} \cong\left\{\left(e^{i n^{2}},()^{n}\right) \mid n \in \mathbf{Z}\right\}$ (which are function algebras on $\Lambda(G)$ and $\mathbf{Z}$, respectively). We remark that these deliberations seem to shed no light on the open question: does it ever happen that the algebra of right distal functions, $\mathcal{D}\left(G^{\prime}\right)$, is not equal to the algebra of left distal functions (analogously defined [5]) for a discrete group $G^{\prime}$ ? It is known that these algebras are not equal for some non-discrete groups, e.g., the euclidean group of the plane $\mathbb{C} \times \mathbb{T}[5 ; 4.6 .17]$.

(b) For this example, we need a discontinuous automorphism $\varphi$ of $\mathbb{T}$ satisfying $\varphi^{2}=1$. [Regard $\mathbb{T}$ as the direct sum of the subgroup $\left\{w \in \mathbf{T} \mid w^{n}=1\right.$ for some $\left.n \in \mathbf{Z}\right\}$ and $c$ copies of $\mathbb{Q}$; then a suitable $\varphi$ just interchanges the coordinates of two fixed copies of $\mathbb{Q}$. A property of $\varphi$ is that, for any $\left(v_{1}, v_{2}\right) \in \mathbb{T} \times \mathbb{T}$, there is a sequence $\left\{u_{n}\right\} \subset \mathbb{T}$ with $u_{n} \rightarrow v_{1}$ and $\varphi\left(u_{n}\right) \rightarrow v_{2}$. See [16, or 11] for more details.] Then $G:=\{\varphi, 1\} \times \mathbf{T}$ with multiplication

$$
(\epsilon, u)(\delta, v)=(\epsilon \delta, \delta(u) v)
$$

is an equicontinuous compact left topological group, so $\mathcal{C}(G) \subset \mathcal{A P}\left(G_{d}\right)$. For $f \in \mathcal{C}(G), R_{(\delta, v)} f$ is in $\mathcal{C}(G)$ if and only if $(\delta, v) \in \Lambda(G)=\{1\} \times \mathbb{T}$ (unless $f$ is constant on each of $\{\varphi\} \times \mathbb{T}$ and $\{1\} \times \mathbb{T}$, in which case $R_{G} f \subset \mathcal{C}(G)$ ). Thus, $\mathcal{C}(G)$ is not right translation invariant. However, pointwise limits of members of $L_{G} f$ are continuous (i.e., the pointwise closure of $L_{G} f$ is pointwise compact in $\mathcal{C}(G)$ ) for every $f \in \mathcal{C}(G)$; using this last fact, one can show that the closure $L_{G}{ }^{-} \subset \mathcal{C}(G)^{\mathcal{C}(G)}$ is a compact topological group antiisomorphic to $\lambda_{G}{ }^{-} \subset G^{G}$, $\lambda_{G}{ }^{-} \cong\{\varphi, 1\} \times \mathbb{T} \times \mathbb{T}$ with multiplication

$$
\left(\epsilon, u_{1}, u_{2}\right)\left(1, v_{1}, v_{2}\right)=\left(\epsilon, u_{1} v_{1}, u_{2} v_{2}\right),\left(\epsilon, u_{1}, u_{2}\right)\left(\varphi, v_{1}, v_{2}\right)=\left(\epsilon \varphi, u_{2} v_{1}, u_{1} v_{2}\right)
$$

$(\epsilon, u) \in G$ corresponding to $(\epsilon, u, \varphi(u)) \in\{\varphi, 1\} \times \mathbf{T} \times \mathbf{T}$. (See [11] for more details about $\lambda_{G}{ }^{-}$.) $L_{G}{ }^{-}$and $\lambda_{G}{ }^{-}$are antiisomorphic because $L_{G}$ and $\lambda_{G}$ are. In the next section (Theorem 8), the relationship of this antiisomorphism to Theorem 3(ii) will be discussed in the context of compact right topological groups.

(c) We have more comments to make about the group $\{\varphi, 1\} \times \mathbf{T}$ of (b), comments not pertaining directly to the present work, but relating to the structure theorem (Theorem 2 above) for compact groups with one-sided continuity and its proof, and to the existence of Haar measure. For these comments, we prefer to discuss the compact right topological group $G=\mathbb{T} \times\{\varphi, 1\}$ with multiplication

$$
(u, \epsilon)(v, \delta)=(u \epsilon(v), \epsilon \delta)
$$

(which is antiisomorphic to $\{\varphi, 1\} \times \mathbb{T}$ ). Now, $\Lambda(G)=\mathbb{T} \times\{1\}$ and is not dense in $G$, so $G$ does not satisfy all of the hypotheses of the structure theorem (Theorem 2) for compact right topological groups. Nonetheless, it obviously satisfies the conclusion: the required system of subgroups is $L_{0}=G, L_{1}=\mathbb{T} \times\{1\}$ with $L_{0} / L_{1} \cong\{\varphi, 1\}$, and $L_{2}=\{e\}$. So, $G$ has Haar measure $\mu$, which is right invariant, unique as such, and also left invariant [12]. One can identify $\mu$ right away in any case; it is just Lebesgue measure on $\mathbb{T}$, divided by two, on each of $\mathbb{T} \times\{1\}$ and $\mathbb{T} \times\{\varphi\}$. It is known that Haar measure on a compact right topological group is not always uniquely determined by left invariance (i.e., by invariance under left translation 
by members of $\Lambda(G)$ ) when $\Lambda(G)$ is dense in $G$ [2]. One verifies readily that $\mu$ is not uniquely determined by left invariance here either; the left invariant probability measures on $\mathbb{T} \times\{\varphi, 1\}$ are just the convex combinations of the Lebesgue measures on $\mathbf{T} \times\{1\}$ and $\mathbf{T} \times\{\varphi\}$.

Since the conclusion of the structure theorem holds, and also since $G$ has such a tractable form, one might expect that the method of proof of the structure theorem would still determine $G$ 's system of subgroups, even though not all of the hypotheses of the structure theorem are satisfied. We show now that this is not the case. Let $\tau$ denote the metric topology that $G$ has to start with. We must discuss the $\sigma$-topology of $G$, which is the quotient topology that $G$ acquires relative to the map

$$
\theta:(s, t) \mapsto s^{-1} t,(G, \tau) \times(G, \tau) \rightarrow G ;
$$

that is, $U \subset G$ is $\sigma$-open if $\theta^{-1}(U)$ is $\tau \times \tau$-open in $G \times G$. (See [15, or 5; Appendix C] for more details on the $\sigma$-topology, noting, in particular, that $\sigma \neq \tau$ [15; Theorem 1.1], and that [15; Theorem 1.2(2)] does not apply, since $\Lambda(G)$ is not dense in $G$; in fact, the sets

$$
\left\{U^{-1} U \mid U \text { is a } \tau \text {-neighbourhood of } e\right\}
$$

form a basis for $\tau$-neighbourhoods of $e$, and hence do not form a basis for $\sigma$-neighbourhoods of $e$.) We obviously have

$$
\theta((u, \varphi),(u, \varphi))=\left(\varphi\left(u^{-1}\right), \varphi\right)(u, \varphi)=\left(\varphi\left(u^{-1}\right) \varphi(u), 1\right)=\left(\varphi\left(u^{-1} u\right), 1\right)=(1,1)=e
$$

and so conclude that every $\sigma$-neighbourhood of $e$ contains a set $A:=(\varphi(V), 1)$, where $V$ is an ordinary neighbourhood of $1 \in \mathbf{T}$. By the property of $\varphi$ asserted near the beginning of Example 4(b), $\varphi(V)$ is dense in $\mathbf{T}$, so $A$ is $\tau$-dense, hence also $\sigma$-dense, in $\mathbf{T} \times\{1\} \subset G$. Since $\mathbf{T} \times\{1\}$ is $\sigma$-open

$$
\left(\theta^{-1}(\mathbb{T} \times\{1\})=(\mathbb{T} \times\{1\})^{2} \cup(\mathbb{T} \times\{\varphi\})^{2}\right),
$$

hence also $\sigma$-closed, the method of proof of the structure theorem does give $L_{1}=\mathbb{T} \times\{1\}$ as the intersection of all $\sigma$-closed $\sigma$-neighbourhoods in $G$ of $e$. The next step in the proof of the structure theorem is to restrict $G$ 's topology $\sigma$ to $L_{1}$ and to get $L_{2}$ as the intersection of all $\sigma$-closed $\sigma$-neighbourhoods in $L_{1}$ of e; however, since every $\sigma$-neighbourhood (in $G$ or in $L_{1}$ ) of $e$ contains a set $A=(\varphi(V), 1) \subset L_{1}$ which is dense in $L_{1}$ (as indicated above), this intersection must be just $L_{1}$ again. So the induction process of the proof of the structure theorem has terminated at $L_{1}$ and does not get down to $\{e\}$, even though $G$ does have the required system of subgroups.

Since the structure theorem holds for every group that is a closed subgroup of a compact right topological group $G^{\prime}$ with dense topological centre $\Lambda\left(G^{\prime}\right)$ [12], one must wonder if $G=\mathbb{T} \times\{\varphi, 1\}$ can be embedded as a closed subgroup of such a $G^{\prime}$. However, this is not the case; in fact, $G$ cannot be embedded in any compact right topological group $G^{\prime}$ for which the proof of the structure theorem "works", i.e., produces a system of subgroups $\left\{L_{\xi}^{\prime}\right\}$ for $G^{\prime}$ as in Theorem 2. For, if $G$ is a subgroup of such a $G^{\prime}$, the proof of the structure theorem must also "work" for $G$ (i.e., produce a system of subgroups for $G$ as in Theorem 2); this is because of the observation $\left[15 ;\right.$ p. 197] that the $\sigma$-topology of $G^{\prime}$ is weaker than the $\sigma$-topology of $G$. 
It is noteworthy that the restriction of the $\sigma$-topology of $G$ to $L_{1}=\mathbb{T} \times\{1\}$ is not the same as the $\sigma$-topology of $L_{1}$ (call it $\left.\sigma_{1}\right)$, which is defined using the map

$$
\theta_{1}:(s, t) \mapsto s^{-1} t, \quad\left(L_{1}, \tau\right) \times\left(L_{1}, \tau\right) \rightarrow L_{1} ;
$$

since $L_{1}=\mathbb{T} \times\{1\} \cong \mathbb{T}$ is a topological group, $\sigma_{1}$ is the same as $\tau$ (i.e., the same as the restriction of $\tau$ to $\left.L_{1}\right)$ [15; Theorem 1.1]. Is this phenomenon $\left(\left.\sigma\right|_{L}\right.$ is not the same as the $\sigma$-topology of $L$ ) possible for a closed subgroup $L$ of a compact right topological group with dense topological centre? We do not know the answer.

The situation is somewhat different for the group $G=\{ \pm 1\} \times \mathbb{T}$ of Example 7.2 below (which also appears in [12]). Again $\Lambda(G)$ is not dense in $G$, since it equals $\{e\}$, but $G$ does not have a system of subgroups, as in the conclusion of the structure theorem. Nonetheless, $G$ does have Haar measure, which obviously is not uniquely determined by left invariance. This group also cannot be embedded in a compact right topological group $G^{\prime}$ with $\Lambda\left(G^{\prime}\right)$ dense in $G^{\prime}$.

\section{THE COMPACT TOPOLOGICAL GROUP FROM $\mathcal{C}(G) \cap \mathcal{A P}\left(G_{\boldsymbol{d}}\right)$.}

Theorem 3 tells us that $\mathcal{C}(G) \subset \mathcal{D}\left(G_{d}\right)$ if and only if $G$ is distal and $\mathcal{C}(G) \subset \mathcal{A P}\left(G_{d}\right)$ if and only if $G$ is equicontinuous. What can be said about $\mathcal{C}(G) \cap \mathcal{D}\left(G_{d}\right)$ when $G$ is not distal, and about $\mathcal{C}(G) \cap \mathcal{A P}\left(G_{d}\right)$ when $G$ is not equicontinuous? At the end of Example 7.2, we make our only comment about $\mathcal{C}(G) \cap \mathcal{D}\left(G_{d}\right)$ (when it not equal to $\mathcal{C}(G)$ ); we have more to say about $\mathcal{C}(G) \cap \mathcal{A P}\left(G_{d}\right)$, and for this topic we prefer again to work with compact right topological groups, for which the notation feels more familiar. So, $G$ denotes a compact right topological group for the rest of this section.

5. LEMMA. Let $G$ be a compact right topological group, and let $\mathcal{F}:=\mathcal{C}(G) \cap \mathcal{A P}\left(G_{d}\right)$. Then $R_{G} f$ is relatively norm-compact in $\mathcal{F}$ and the closure $R_{G}{ }^{-} \subset \mathcal{F}^{\mathcal{F}}$ is a compact topological group.

PROOF. First let us show that, for $f \in \mathcal{F}$, the pointwise closure $R_{G} f^{-} \subset \mathcal{F}$ is norm-compact. For, if $R_{s_{\alpha}} f \rightarrow g \in l^{\infty}\left(G_{d}\right)$ pointwise on $G$, then each $R_{\boldsymbol{s}_{\alpha}} f$ is continuous and the convergence of $\left\{R_{s_{\alpha}} f\right\}$ to $g$ must be uniform, since $f \in \mathcal{A P}\left(G_{d}\right)$; so $g \in \mathcal{F}$. Thus $R_{G}$, restricted to $\mathcal{F}$, is an "almost periodic group of operators", and the desired conclusion follows from the work of de Leeuw and Glicksberg [6] (or see [5; especially chapter 6]).

We remind the reader that, when $\Lambda(G)$ is dense in $G$, the proof of the structure theorem gives the first subgroup $L_{1}$ (of the conclusion of the theorem) as $N(G)$, the smallest kernel of a continuous homomorphism of $G$ into a compact topological group [15]; in particular, $G / L_{1}$ is a compact topological group.

6. THEOREM. Let $G$ be a compact right topological group with $\Lambda(G)$ dense in $G$ and subgroup $L_{1}=N(G)$ from the structure theorem (as indicated above). If $\mathcal{F}=\mathcal{C}(G) \cap \mathcal{A P}\left(G_{d}\right)$, then

$$
\mathcal{F}=\left\{f \in \mathcal{C}(G) \mid f \text { is constant on the cosets } s L_{1} \text { of } L_{1}\right\} \cong \mathcal{C}\left(G / L_{1}\right) .
$$

PROOF. We identify the last two sets of functions [15], and claim that $\mathcal{F} \supset \mathcal{C}\left(G / L_{1}\right)$, because $G / L_{1}$ is a compact topological group and the homomorphism $s \mapsto s L_{1}, G \rightarrow G / L_{1}$, is continuous. To get the reverse containment, we note first that, if $s_{\alpha} \rightarrow s$ in the proof of the last lemma, 
the limit function $g$ equals $R_{s} f$ at all points of $\Lambda(G)$, a dense subset of $G$; since both $g$ and $R_{s} f$ are continuous, they are equal everywhere. A similar (but easier) argument shows that $\mathcal{F}$ is left translation invariant, and so $\mathcal{F}$ is left $m$-introverted. By a standard result (appearing in [5: 4.3.9], for example), the spectrum $X_{\mathcal{F}}$ of $\mathcal{F}$ is a compact topological group. We may think of $G$ as the spectrum of $\mathcal{C}(G)$; then the restiction map $\mathbf{r}:\left.s \mapsto s\right|_{\mathcal{F}}$ is a continuous homomorphism of $G$ onto $X_{\mathcal{F}}$, so the kernel $\mathbf{r}^{-1}(e)$ of $\mathbf{r}$ contains $L_{1}=N(G)$, and $\mathcal{F} \subset \mathcal{C}\left(G / L_{1}\right)$, as required.

7. EXA MPLES. The examples provide further evidence of the great difference that having a non-dense topological centre $\Lambda(G)$ can make.

1. The compact right topological group $G=\mathbb{T} \times\{\varphi, 1\}$ of Example 4(c) has

$$
\mathcal{C}(G) \cap \mathcal{A P}\left(G_{d}\right)=\mathcal{C}(G)
$$

while $\mathcal{C}\left(G / L_{1}\right) \cong \mathcal{C}(\{\varphi, 1\})$ consists of those functions that are constant on each of $\mathbb{T} \times\{1\}$ and $\mathbb{T} \times\{\varphi\}$. Note also that $R_{G} f$, while relatively compact in $\mathcal{C}(G)$ (Lemma 5 ), need not be closed in $\mathcal{C}(G)$; for, using the property of $\varphi$ stated in Example 4(b), we can arrange convergence

$$
R_{\left(v_{n}, 1\right)} f(u, \epsilon)=f\left(u \epsilon\left(v_{n}\right), \epsilon\right) \rightarrow f_{1}(u, \epsilon):=\left\{\begin{array}{l}
f(u, 1), \epsilon=1, \\
f(u v, \varphi), \epsilon=\varphi
\end{array}\right.
$$

Then $f_{1} \notin R_{G} f$ (unless $f(\cdot v, \varphi)=f(\cdot, \varphi)$ ).

2. It is of interest to look at $\mathcal{F}=\mathcal{C}(G) \cap \mathcal{A P}\left(G_{d}\right)$ for a group $G$ that is not distal and for which $\Lambda(G)$ is not dense. Let $G$ be the semidirect product $\{ \pm 1\} \times \mathbb{T}$ with multiplication

$$
(\epsilon, u)(\delta, v)=\left(\epsilon \delta, u^{\delta} v\right)
$$

Give $G$ the topology $\tau$ for which a typical basic neighbourhood of $\left(1, e^{a a}\right)$ or $\left(-1, e^{\imath b}\right)$, where $a<b$, is

$$
A:=\left\{\left(1, e^{\imath a}\right),\left(-1, e^{\imath b}\right)\right\} \cup\left\{\left(\epsilon, e^{\imath \theta}\right) \mid \epsilon= \pm 1, a<\theta<b\right\} ;
$$

these basic neighbourhoods are open and closed. Then $(G, \tau)$ is a non-distal compact right topological group and $\Lambda(G)=\{(1,1)\}$ [11; Example 6(d)]. Let us show that $\mathcal{F}$ consists of those $f \in \mathcal{C}(G)$ for which there exists an $f^{\prime} \in \mathcal{C}(\mathbb{T})$ such that $f(\epsilon, u)=f^{\prime}(u)$ for all $(\epsilon, u) \in G$. Suppose that $f \in \mathcal{F}$ and $v_{n}=e^{i / n}$ for $n \in \mathbb{N}$. Then

$$
R_{\left(1, v_{n}\right)} f(\epsilon, u)=f\left(\epsilon, u v_{n}\right) \rightarrow f_{1}(\epsilon, u):=f(1, u)
$$

for all $(\epsilon, u) \in G$, so $f_{1} \in \mathcal{F}$ (Lemma 5); $f_{1}$ can be right translated back to $f$ only if $f(1, u)=$ $f(-1, u)$ for all $u \in \mathbb{T}$ and $f^{\prime}(u):=f(1, u)$ defines an $f^{\prime} \in \mathcal{C}(\mathbb{T})$. Conversely, if $f$ is defined on $G$ like this by means of an $f^{\prime} \in \mathcal{C}(\mathbb{T})$, then $f \in \mathcal{C}(G)$ and $R_{(\delta, v)} f(\epsilon, u)=f^{\prime}\left(u^{\delta} v\right)$, which makes it clear that $R_{G} f \subset \mathcal{F}$, that $h \in R_{G} f$ if and only if the corresponding $h^{\prime} \in \mathcal{C}(\mathbb{T})$ has the form $R_{v} f^{\prime}$ or $R_{v} \check{f}^{\prime}$ for some $v \in \mathbb{T}$ (where $\check{f}^{\prime}(u)=f^{\prime}\left(u^{-1}\right)$ ), and also that $R_{G} f$ contains all its pointwise limits. This last conclusion, which always holds in the setting of Theorem 6 (i.e., when the topological centre is dense), seems strange for this group. The compact topological group $R_{G}{ }^{-} \subset \mathcal{F}^{\mathcal{F}}$ (Lemma 5) is just (isomorphic to the same algebraic group) $\{ \pm 1\} \times \mathbb{T}$ 
furnished with the product topology. Thus, no new points are added to get $R_{G}{ }^{-}$from $R_{G}$. This also seems strange for this group.

The difference between right and left orbits here is noteworthy. Let $f \in \mathcal{F}$ still correspond to $f^{\prime} \in \mathcal{C}(\mathbb{T})$, as above. Then

$$
L_{(\epsilon, u)} f(\delta, v)=f\left(\epsilon \delta, u^{\delta} v\right)=\left\{\begin{array}{l}
R_{u} f^{\prime}(v), \delta=1, \\
R_{u^{-1}} f^{\prime}(v), \delta=-1
\end{array}\right.
$$

so, for $f(\delta, v)=f^{\prime}(v)=v$ (for example), we have

$$
R_{G} f \cap L_{G} f=\mathcal{C}(G) \cap L_{G} f=\{f\}
$$

For this group, we have $\mathcal{F}\left(:=\mathcal{C}(G) \cap \mathcal{A P}\left(G_{d}\right)\right)=\mathcal{C}(G) \cap \mathcal{D}\left(G_{d}\right)$ as well, since we can conclude for $f \in \mathcal{C}(G) \cap \mathcal{D}\left(G_{d}\right)$ (as above for $f \in \mathcal{F}$ ) that

$$
R_{\left(1, v_{n}\right)} f(\epsilon, u) \rightarrow f_{1}(\epsilon, u):=f(1, u)
$$

and $f_{1}$ cannot be right translated back to $f$, unless $f(1, u)=f(-1, u)$ for all $u \in \mathbb{T}$; so $f$ corresponds to an $f^{\prime} \in \mathcal{C}(\mathbb{T})$ and $f \in \mathcal{F}$.

Our final theorem clarifies the relationship of the antiisomorphism mentioned at the end of Example 4(b) to Theorem 3(ii). This is done in the context of compact right topological groups, and can be presented most effectively if we use right notation for the right translation flow $\left(G,{ }_{G} \rho\right),{ }_{s} \rho: t \mapsto(t)_{s} \rho:=t s$ (as in [11]).

8. THEOREM. For a compact right topological group $G$ the following assertions are equivalent:

(i) $G$ is equicontinuous, i.e., the right translation flow $\left(G,{ }_{G} \rho\right)$ is equicontinuous.

(ii) The closure ${ }_{G} \rho^{-} \subset G^{G}$ is a compact topological group.

(iii) $\mathcal{C}(G) \subset \mathcal{A P}\left(G_{d}\right)$.

(iv) $R_{G}{ }^{-} \subset \mathcal{C}(G)^{\mathcal{C}(G)}$ is a compact topological group.

When these conditions hold, ${ }_{G} \rho^{-} \subset G^{G}$ is isomorphic to $R_{G}{ }^{-} \subset \mathcal{C}(G)^{\mathcal{C}(G)}$.

PROOF. We assert the equivalence of (i) and (ii). The equivalence of (i) and (iii) is just Theorem 3 in its other-sided form. Lemma 5 tells us that (iii) implies (iv). If (iv) holds and $f \in \mathcal{C}(G)$, then the function $T \mapsto T f$ maps $R_{G}{ }^{-}$continuously into $\mathcal{C}(G)$, and in fact, onto the closure in $\mathcal{C}(G)$ of the orbit $R_{G} f$ of $f$; so this closure is norm-compact, $f \in \mathcal{A P}\left(G_{d}\right)$, and (iii) is satisfied.

When (i) - (iv) are satisfied, the isomorphism between ${ }_{G} \rho^{-}$and $R_{G}{ }^{-}$that we want is just the continuous extension to ${ }_{G} \rho^{-}$of the homomorphism $\psi:{ }_{s} \rho \mapsto R_{s},{ }_{G} \rho \rightarrow R_{G}$. So, suppose that ${ }_{{ }_{\alpha}} \rho \rightarrow \mu \in G^{G}$, the convergence being uniform on $G$. An $f \in \mathcal{C}(G)$ and an $\epsilon>0$ determine a member $L_{f,+}$ of the uniformity of $G$,

$$
U_{f, \epsilon}:=\left\{\left(s_{1}, s_{2}\right) \in G \times G|| f\left(s_{1}\right)-f\left(s_{2}\right) \mid<\epsilon\right\}
$$

[10]. from which it follows that $\left\{R_{\boldsymbol{s}_{\alpha}} f\right\}$ is a Cauchy net in $\mathcal{C}(G)$. Hence, $\left\{R_{\boldsymbol{s}_{\alpha}}\right\}$ is Cauchy in $\mathcal{C}(G)^{C(G)}$. This shows that $\psi$ extends continuously to ${ }_{G} \rho^{-}$. We complete the proof by showing that the extension of $\psi$ is one-to-one. Suppose that $s_{\alpha} \rho \rightarrow \mu$ as above and that $\mu \neq c \in{ }_{G} \rho^{-}$. 
Then $(s) \mu \neq s$ for some $s \in G$, and there is an $f \in \mathcal{C}(G)$ such that $f((s) \mu) \neq f(s)$. It follow's that

$$
\lim _{\alpha} R_{s_{\alpha}} f(s)=\lim _{\alpha} f\left(s s_{\alpha}\right)=f((s) \mu) \neq f(s),
$$

so $\lim _{\alpha} R_{\boldsymbol{s}_{\alpha}} f \neq f$ and $\psi(\mu):=\psi\left(\lim _{\alpha} \boldsymbol{s}_{\alpha} \rho\right)=\lim _{\alpha} \psi\left(\boldsymbol{s}_{\alpha} \rho\right)=\lim _{\alpha} R_{\boldsymbol{s}_{\alpha}} \neq e \in R_{G}^{-}$.

The reader will have noted the powerful blend of left and right notations required to got the compact topological groups to be isomorphic in the last statement of the theorem. One way of getting an isomorphism in the other-sided setting (e.g., in Example 4(b)) would be to use right notation for the left translation operator, $[(f), L](t)=f(s t)$ (which looks horrible. although it is merely the other-sided form of writing right translation operators on the left, as we have done throughout this paper). The reason we would then get an isomorphism is that $s \mapsto s$ is a homomorphism, as is $s \mapsto R_{s}$, while $s \mapsto L$, is an antihomomorphism.

ACKNOWLEDGEMENT. This work was supported in part by NSERC grant A7857.

\section{REFERENCES}

1. ANZAI, H. and S. KAKUTANI, Bohr compactifications of a locally compact abelian group I, II, Proc. Imp. Acad. Tokyo 19 (1943), 476-480, 533-539.

2. AUSLANDER, L. and F. HAHN, Real functions coming from flows on compact spaces and concepts of almost periodicity, Trans. Amer. Math. Soc. 106 (1963), 415-426.

3. BAKER, J.W. and P. MILNES, The ideal structure of the Stone-Cech compactification of a group, Math. Proc. Camb. Phil. Soc. 82 (1977), 401-407.

4. BERGLUND, J.F. and K.H. HOFMANN, Compact Semitopological Semigroups and Weakly Almost Periodic Functions, Lecture Notes in Mathematics 42, Springer-Verlag, New York, 1967.

5. BERGLUND, J.F., H.D. JUNGHENN and P. MILNES, Analysis on Semigroups: Function Spaces, Compactifications, Representations, Wiley, New York, 1989.

6. DE LEEUW, K. and I. GLICKSBERG, Applications of almost periodic compactifications, Acta Math. 105 (1961), 63-97.

7. ELLIS, R. Distal transformation groups, Pacific J. Math. 9 (1958), 401-405.

8. ELLIS, R. Lectures on Topological Dynamics, Benjamin, New York, 1969.

9. FURSTENBERG, H. The structure of distal flows, Amer. J. Math. 85 (1963), 477-515.

10. KELLEY, J.L. General Topology, Van Nostrand, Princeton, N.J., 1955.

11. MILNES, P. Distal compact right topological groups (to appear in Acta Math. Hung.)

12. MILNES, P. and J. PYM, Haar measure for compact right topological groups, Proc. Amer. Math. Soc. 114 (1992), 387-393.

13. MILNES, P. and J. PYM, Homomorphisms of minimal and distal flows (to appear in $\underline{J}$. Nigerian Math. Soc.)

14. MITCHELL, T. Topological semigroups and fixed points, Illinois J. Math. 14 (1970), 630-641.

15. Namioka, I. Right topological groups, distal flows and a fixed point theorem, Math. Systems Theory 6 (1972), 193-209.

16. Ruppert, W.A.F. Über kompakte rechtstopologische Gruppen mit gleichgradig stetigen Linkstranslationen, Sitzungsberichten der Österreichischen Akademie der Wissenschaften Mathem.-naturw. Klasse, Abteilung II 184 (1975), 159-169. 


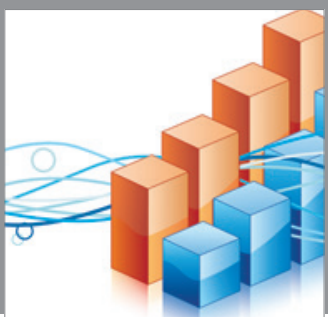

Advances in

Operations Research

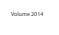

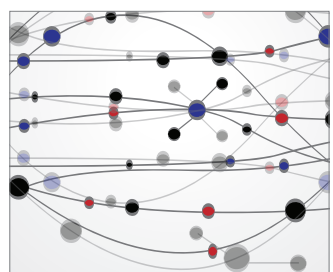

\section{The Scientific} World Journal
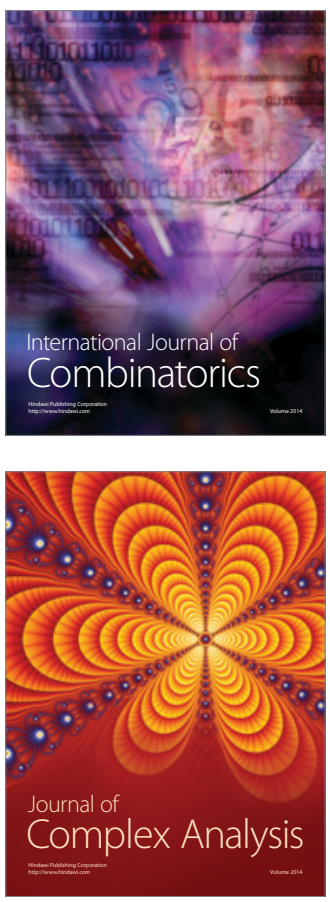

International Journal of

Mathematics and

Mathematical

Sciences
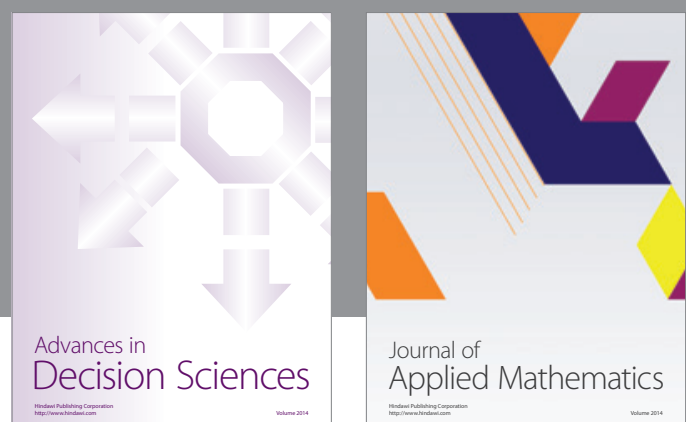

Journal of

Applied Mathematics
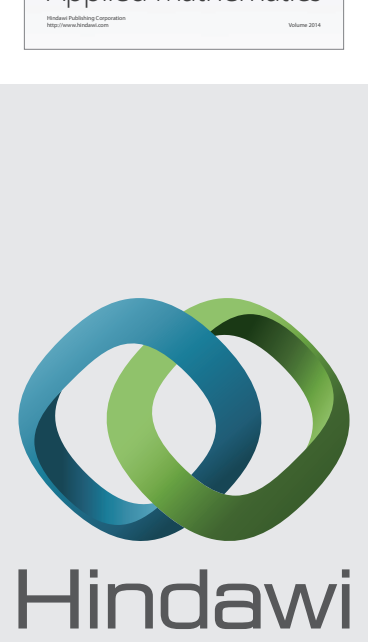

Submit your manuscripts at http://www.hindawi.com
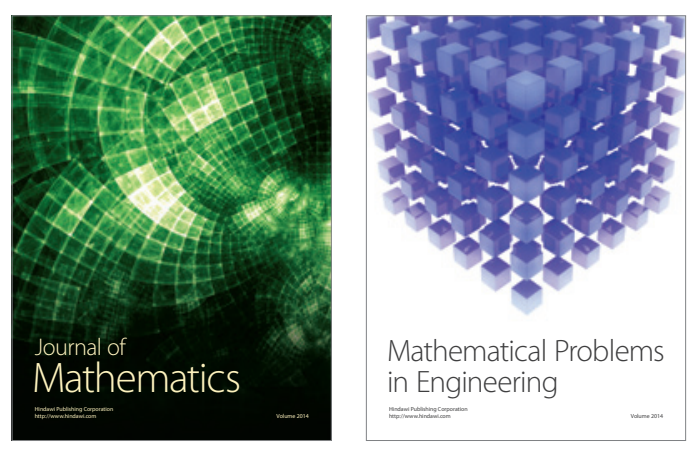

Mathematical Problems in Engineering
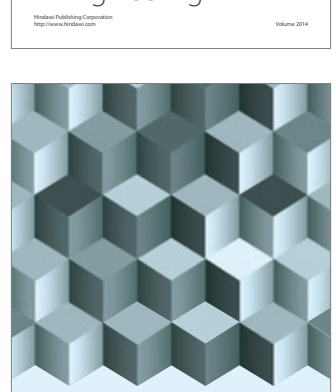

Journal of

Function Spaces
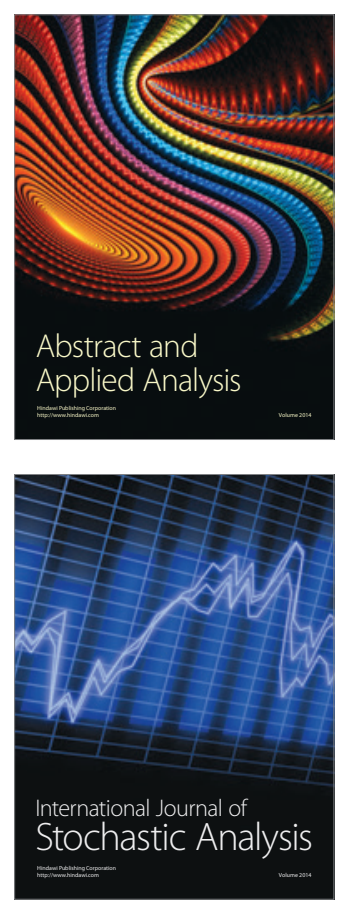

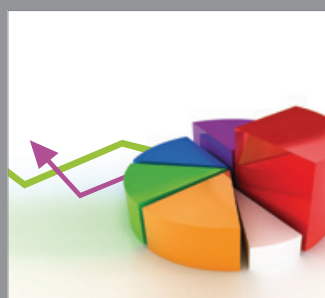

ournal of

Probability and Statistics

Promensencen
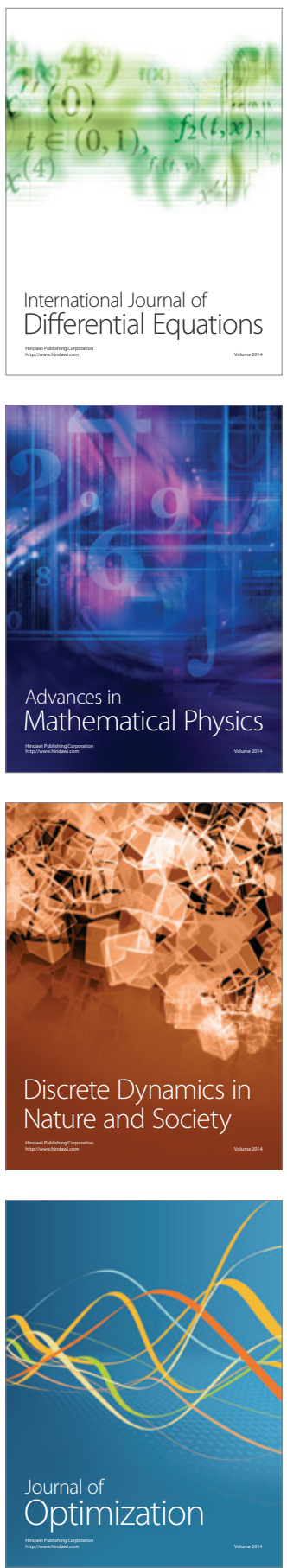